Pacific Journal of Mathematics

INJECTIVE MODULES OVER DUO RINGS 


\title{
INJECTIVE MODULES OVER DUO RINGS
}

\author{
Thomas S. SHORES
}

\begin{abstract}
Let $R$ be a ring with unit whose right and left ideals are two-sided ideals. It is shown that every Noetherian injective $R$-module has finite length (i.e., has a finite composition series). If $I$ is a maximal ideal of $R$, then $R$ has a universal localization, $R_{I}$ at $I$. The condition that the injective hull of $R / I$ is finite is characterized in terms of $R_{I}$.
\end{abstract}

1. Introduction. In this note all rings have unit and modules are unital right modules. A. Rosenberg and D. Zelinsky have shown that if $R$ is a commutative ring and $I$ is a maximal ideal of $R$, then the injective hull of $R / I$ is finite (i.e., has finite length) if and only if the localization of $R$ at $I$ is Artinian (see Theorem 5 of [6, p. 379]). In this note we shall prove an extended version (Theorem 4) of their result for a class of rings which is somewhat interesting in itself. Let us call a ring $R$ a duo ring if $x R=R x$ for all $\mathrm{x} \in R$ (equivalently all ideals are bilateral). Such rings were investigated by E. Feller [2] and G. Thierrin [7]. Trivial examples of duo rings are, of course, commutative rings and division rings. Nontrivial duo rings are not difficult to come by (e.g., any noncommutative special primary ring is duo, since the only right or left ideals are powers of the unique maximal ideal). In fact some interesting examples of duo rings have already occurred in the literature: M. Auslander and O. Goldman have shown in $[1$, p. 13] that there exist noncommutative maximal orders which are both duo rings and Noetherian domains. Further investigations of such rings have been carried out by G. Maury in [4].

One of the basic difficulties in extending Rosenberg and Zelinsky's result to duo rings is the existence of suitable localizations. This problem is considered in $\S 2$. Next we show in $\S 3$ that Noetherian duo rings are classical in the sense that the familiar primary decomposition theory of commutative Noetherian rings extends to duo rings. We use this fact to show that Noetherian injective modules over duo rings are finite. Finally we prove our main result in $\S 4$.

The injective hull of the module $M$ will be denoted by $E(M)$. If $A$ and $B$ are subsets of $M$ or $R$, then $A \cdot B=\{x \in R \mid x B \subseteq A\}$ and $A \cdot B=\{x \in R \mid A x \subseteq B\}$. Also $A \backslash B$ is the set of elements in $A$ but not $B$.

2. Localizations. First of all we need a suitable definition of the term "localization." The ideal $P$ of $R$ is prime if $A B \cong P$ implies $A \subseteq P$ or $B \subseteq P$ for all ideals $A$ and $B$ of $R$. 
Definition 1. Let $R$ be a ring and $P$ a prime ideal of $R$. By a (right) localization of $R$ at $P$ we mean a nonzero ring $Q$ together with a ring homomorphism $\phi: R \rightarrow Q$ such that images under $\phi$ of elements of $R \backslash P$ (elements in $R$ but not $P$ ) are units in $Q$ and $Q=$ $\left\{\phi(a) \phi(b)^{-1} \mid a \in R\right.$ and $\left.b \in R \backslash P\right\}$.

REMARK 1. A localization $Q$ of $R$ at $P$ is a local ring whose maximal ideal is $\phi(P) Q$. For if $\phi(a) \phi(b)^{-1}$ is a unit of $Q$, then so is $\phi(a)$, say $\phi(a) \phi(c) \phi(d)^{-1}=1$. But then $a c-d \in \operatorname{ker} \phi \subseteq P$, so that $a \notin P$. Hence the units of $Q$ are precisely the elements of the set $\phi(R \backslash P) \phi(R \backslash P)^{-1}$ and the non-units form a maximal right ideal $\phi(P) Q$. Therefore $Q$ has a unique maximal right ideal and is by definition a local ring.

Definition 2. The localization $Q$ of $R$ at $P$ is universal if every localization $\phi^{*}: R \rightarrow Q^{*}$ of $R$ at $P$ can be factored through $\phi: R \rightarrow Q$, i.e., there is a ring homomorphism $\lambda: Q \rightarrow Q^{*}$ such that $\lambda \cdot \phi=\phi^{*}$.

Clearly universal localizations are unique up to a ring isomorphism, if they exist. If $R$ has a universal localization at $P$, we shall denote this ring by $R_{P}$. In the case of a commutative ring $R, R_{P}$ exists and is just the usual ring of quotients of $R$ by elements of $R \backslash P$. With some slight modifications, we can make the same trick work for duo rings:

Theorem 1. If $P$ is a prime ideal of the duo ring $R$, then $R$ has a universal localization at $P$.

Proof. First note that if $x, y \in R$ and $x y \in P$, then $x R y R=x y R \subseteq$ $P$, so $x \in P$ or $y \in P$. Hence $R \backslash P$ is multiplicatively closed. Let $K$ be the set of $r \in R$ for which there are elements $a, b \in R \backslash P$ such that $a r b=0$. Then $K$ is an ideal of $R$. For if $a r b=0$ and $a^{\prime} r^{\prime} b^{\prime}=0$, then $a a^{\prime}\left(r-r^{\prime}\right) b b^{\prime}=0$. To see this apply the fact that $x R=R x$ for all $x \in R$ to obtain that $a a^{\prime} r b b^{\prime}=a^{*} a r b b^{\prime}=0$ for some $a^{*} \in R$. Similarly $a a^{\prime} r^{\prime} b b^{\prime}=0$ and as $\in K$ if $s \in R$. If $r \in R$ and $a, b \in R \backslash P$ are such that $a r b \in K$, then $a^{\prime} a r b b^{\prime}=0$ for suitable $a^{\prime}, b^{\prime} \in R \backslash I$. Hence $r \in K$. Hence $r \in K$. In other words images of elements of $R \backslash I$ in the ring $R / K$ are regular elements (i.e., nonzero divisors). Let $S=$ $(R / K) \backslash(P / K)$. Since $R / K$ is a duo ring, it follows that for any $u \in$ $R / K$ and $v \in S$ there is an element $w \in R / K$ such that $u v=v w$. Thus elements of $S$ satisfy a right Ore condition, so we may form the ring of right quotients $Q$ of $R / K$ by elements of $S$ in the usual way (i.e., $Q$ is the set of equivalence classes of ordered pairs $(a, b)$ such that $b \notin P$ and $(a, b)$ is equivalent to $(c, d)$ if $d a=e b$, where $d c=e d)$. If we identify $R / K$ with its image in $Q$, then it is clear that $Q$, along with the natural map $\phi: R \rightarrow R / K$, is a right localization of $R$ at $P$. 
To see that $Q$ is universal, simply note that if $\phi^{*}: R \rightarrow Q^{*}$ is any other localization of $R$ at $P$, then $Q^{*}$ is determined up to a ring isomorphism by $\operatorname{Ker} \phi^{*}$. For $Q^{*}$ is just the ring of right quotients of $R / \operatorname{ker} \phi^{*}$ by elements of $S^{*}=\left(R / \operatorname{ker} \phi^{*}\right) \backslash\left(P / \operatorname{ker} \phi^{*}\right)$. However, it is clear that $K \cong \operatorname{ker} \phi^{*}$ and this implies that the ring of right quotients of $R / \operatorname{ker} \phi^{*}$ by $S^{*}$ is just $Q /\left(\left(\operatorname{ker} \phi^{*}\right) Q\right)$, for

$$
R / \operatorname{ker} \phi^{*} \cong(R / K)\left(\operatorname{ker} \phi^{*} / K\right) \text {. }
$$

REMARK 2. In the case of commutative $R$, the ring $R_{P}$ is just the usual localization and is itself a commutative ring. It is not clear that $R_{P}$ need be a duo ring if $R$ is a duo ring. We leave this question open. The ideal $K$ of the proof of Theorem 1 will be called the $P$ component of 0 . The set of $r \in R$ for which $r b=0$ for some $b \in R \backslash P$ will be called the right P-component of 0 . Of course if $R$ is commutative, then the right $P$-component of 0 is just $K$.

3. Noetherian duo rings. Let $A$ be an ideal of the ring $R$. Then by the radical of $A$ we mean the inverse image in $R$ of the prime radical of the ring $R / A$. As usual we call the ideal $A$ of $R$ a primary ideal if the radical of $A$ is a prime ideal and whenever $C$ and $D$ are ideals of $R$ such that $C D$ or $D C$ is contained in $A$ and $C \varsubsetneqq$ $A$, then $D$ is contained in the radical of $A$. Also $A$ is called an irreducible ideal if $A$ cannot be written as the intersection of ideals of $R$ which properly contain $A$. We need to extend the well known fact that irreducible implies primary in Noetherian commutative rings.

Lemma 1. Let $P$ be the prime radical of the Noetherian duo ring $R$ and suppose that 0 is an irreducible ideal of $R$. Then elements of $R \backslash P$ are regular.

Proof. The ideal $P$ is nilpotent by Levitzki's Theorem, since $R$ is Noetherian. Furthermore if $r^{n}=0$ for some $r \in R$, then $(r R)^{n}=r^{n} R=0$ since $R$ is a duo ring. Therefore $P$ consists precisely of the nilpotent elements of $R$. Suppose that $r \in R \backslash P$ and $0 \neq a \in R$ are such that $a r=$ 0 . Then $r^{n} \notin P$ for all integers $n$. Apply the fact that $R$ is Noetherian to the ascending chain $0 \cdot r \subseteq 0 \cdot r^{2} \subseteq \cdots$ to obtain that $0 \cdot r^{n}=0 \cdot r^{n+1}$ for some integer $n$. Let $d \in R r^{n} \cap R a$, say $d=s r^{n}=s^{\prime} a$. Then $s r^{n+1}=$ $s^{\prime} a r=0$, so $s \in 0 . r^{n+1}=0 .^{\bullet} r^{n}$ and $s r^{n}=0=d$. Hence $0=R r^{n} \cap R a$, which contradicts the fact that 0 is irreducible. Hence $r$ is right regular. Repeat the preceding argument on the chain $r \cdot .0 \subseteq r^{2} \cdot .0 \subseteq \cdots$ and obtain that $r$ is left regular (and therefore regular).

COROLlaRy. Every irreducible ideal of a Noetherian duo ring $R$ is a primary ideal. 
Proof. Let $A$ be an irreducible ideal of $R$. Without loss of generality $A=0$. If $P$ is the prime radical of $R$, then $P$ is a prime ideal; for if $x, y \in R \backslash P$ and $x y \in P$, then there is a least integer $n$ such that $(x y)^{n} \neq 0$. Then $(x y)^{n+1}=0$, which implies that $x$ or $y$ is not regular. This contradicts Lemma 1 , so $P$ is a prime ideal. Likewise apply Lemma 1 and obtain that if $x y$ or $y x$ equals 0 and $x \neq 0$, then $y \in P$. Hence 0 is a primary ideal.

A standard argument (see Exercises 9-13 of [3, p. 105]) shows that every ideal in a Noetherian ring can be expressed as a finite intersection of irreducible ideals. Let $R$ be a duo ring and collect all the irreducibles in such an expression whose radical is the same prime ideal. Then we obtain the following result:

Theorem 2. Let $A$ be an ideal in the Noetherian ideal ring $R$. Then $A$ is the intersection of a finite number of irreducible ideals, no two of which have the same radical.

The proof of the next result provides us with some information about injective modules over Noetherian duo rings which we shall use in Theorem 4. This proof uses Lemma 1 and some results and techniques of E. Matlis [5] for injective modules.

THEOREM 3. Every Noetherian injective module over a duo ring has finite length.

Proof. Let $R$ be a duo ring and $M$ an injective Noetherian $R$ module. Let $K$ be the (right) annihilator of $M$ in $R$. Then $R / K$ is a Noetherian ring, since $R / K$ is a subdirect sum of $R$-modules $R / K_{i}$, $i=1, \cdots, m$, where $K_{i}$ is the annihilator of $x_{i}$ in $R$ and $M=x_{1} R+$ $\cdots+x_{m} R$. Also $R / K$ is a duo ring. Furthermore $M$ is certainly injective as an $(R / K)$-module, so we may as well replace $R$ by $R / K$ and assume that $K=0$ and $R$ is Noetherian. Thus $M$ is a finite direct sum of indecomposible injective submodules by Theorem 2.5 of [5, p. 516]. We may replace $M$ by one of these summands and assume that $M$ is itself indecomposible.

Now apply Theorem 2.4 of [5, p. 516] and we obtain that $M=$ $E(R / J)$, where $J$ is an irreducible ideal of $R$. Furthermore if $0 \neq$ $x \in M$, then $M \cong E(R /(x \cdot 0))$ by the same theorem. Let $P$ be the radical of $J$. Then there is a least integer $n$ such that $P^{n+1} \cong J$, since $R$ is Noetherian. Select an element $y \in P^{n} \backslash P^{n+1}$ and obtain from the above remarks that $M \cong E(R /(y \cdot . J))$. Clearly $P \subseteq y \cdot . J$. Also if $a \in y \cdot . J$, then $y a \in J$. Since images of elements of $R \backslash P$ are regular in $R / J$ by Lemma $1, a \in P$. Therefore $y \cdot . J=P$, which is a prime ideal by the Corollary to Lemma 1 . Furthermore $M \cong E(R / P)$ by a 
preceding remark. Now any prime duo ring is an integral domain, for $x y=0$ implies $x R y R=0$. Furthermore a prime duo ring obviously satisfies an Ore condition, so such a ring is contained in a right quotient division ring. Let $Q$ be the right quotient ring of $R / P$. The injection of $R / P$ into $M$ extends to a $R$-homomorphism of $Q$ into $M$. Such a homomorphism is clearly injective, so $Q$ is a submodule of $M$ and therefore Noetherian. If $x \neq 0$, we obtain from $x R=R x$ that $x^{-1} R=R x^{-1}$. Hence any element $a^{\prime} b^{-1}$ of $Q$ may be written as $b^{-1} a$ for suitable $a$. In particular there are elements $a_{i}, b_{i} \in R, i=1, \cdots, n$, such that

$$
Q=b_{1}^{-1} a_{1}(R / P)+\cdots+b_{n}^{-1} a_{1}(R / P) .
$$

But then $Q=b_{1} b_{2} \cdots b_{n} Q$ and repeated application of the identity $x^{-1} R=R x^{-1}$ yields that $b_{1} \cdots b_{n} b_{i}^{-1} a_{i}=b_{1} \cdots b_{i-1} c a$ for some $c \in R$. Consequently $Q=R / P$ and $P$ is actually a maximal ideal of $R$.

We complete the proof by showing that $P^{n}=0$ for some integer $n$. For it then follows that $R$ is a local Noetherian ring with nilpotent maximal ideal $P$. Since $P^{i} / P^{i+1}$ must be a finite dimensional $(R / P)$ vector space for all integers $i$, one obtains that $R$ is (right) Artinian. Consequently $M$ must be Artinian, which was to be shown. Now if $P_{1}$ is any other prime ideal of $R$ and $E\left(R / P_{1}\right) \cong E(R / P)$, then we can think of $R / P_{1}$ and $R / P$ as embedded in $E(R / P)$ and obtain that $R / P_{1} \cap$ $R / P \neq 0$. But nonzero elements of $R / P_{1}$ have right annihilator $P_{1}$ in $R$ and likewise for nonzero elements of $R / P$. Hence $P=P_{1}$. It follows from the remarks in the previous paragraph that if $0 \neq x \in M$, then $P^{m} \cong x \cdot .0$ for some integer $m$. Form the ascending chain of submodules of $M$ given by $A_{i}=\left\{x \in M \mid x P^{i}=0\right\}, i=1,2, \cdots$. Then there is an integer $n$ such that $A_{n}=A_{n+1}$, since $M$ is Noetherian. But we have shown that $M$ is the union of the $A_{i}, i=1,2, \cdots$. Hence $M=A_{n}$ and $M P^{n}=0$. But $M$ is a faithful $R$-module, so $P^{n}=$ 0 and the proof is complete.

3. The main theorem. Note that if $I$ is a maximal right ideal of the duo ring $R$, then $I$ is an ideal of $R$ and $R / I$ is a division ring, so $R_{I}$ exists. We now generalize Rosenberg and Zelinskys' result. First we need the following lemma:

Lemma 2. Let $R$ be a duo ring with a maximal ideal $I$ and let $M$ be a nonzero $R$-module with the property that right multiplication of $N$ by an element of $R \backslash I$ is a one-to-one map of $N$ onto $N$ for all submodules $N$ of $M$. Then $M$ can be made into an $R_{I}$-module in such a way that $R$ - and $R_{I}$-submodule lattices of $M$ coincide.

Proof. Let $K=M \cdot 0$. Then $M$ is a faithful $(R / K)$-module. Also 
$(R / K)_{I}=R_{I} / K R_{I}$, since $K \subseteq I$. So it is sufficient to prove the lemma in the case that $K=0$ and we may assume that $M$ is a faithful $R$ module. Right multiplication by an element of $R \backslash I$ is a one-to-one map of $N$ onto $N$, where $N$ is any submodule of $M$. In particular if $a r=0$ with $a \in R$ and $r \in R I$, then $(M a) r=0$. But $M a R=M R a=M a$, since $R$ is a duo ring. Hence $M a$ is a submodule of $M$ and therefore $M a=0$. Since $M$ is a faithful $R$-module, $a=0$. Similarly if $r a=0$, then $M r a=M a=0$ and $a=0$. Hence elements of $R \backslash I$ are regular elements of $R$. Therefore $R$ is actually a subring of $R_{I}$. So if $r \in R \backslash I$, we obtain from $r R=R r$ that $r^{-1} R=R r^{-1}$.

Since right multiplication by an element of $R \backslash I$ is a one-to-one map of $M$ onto $M$, we can define, for all $m \in M$ and $r \in R I, m r^{-1}=$ $m^{\prime}$, where $m^{\prime} r=m$. For any $a \in R$ define $m\left(a b^{-1}\right)=(m a) b^{-1}$. If $a b^{-1}=$ $c d^{-1}$, then we can select an element $e \in R$ such that $d^{-1} b=e d^{-1}$, since $d^{-1} R=R d^{-1}$. Thus we obtain equations

$$
\left(m\left(a b^{-1}\right)\right)(b d)=m a d, \text { and } m\left(c d^{-1}\right)(d e)=m c e .
$$

But $a d=c e$, so we obtain from these equations that $m\left(a b^{-1}\right)=$ $m\left(c d^{-1}\right)\left(d e d^{-1} b^{-1}\right)$. Since $d e d^{-1} b^{-1}=1$, we conclude that $m\left(a b^{-1}\right)=m\left(c d^{-1}\right)$ and multiplication by elements of $R_{I}$ is well-defined. Similarly it follows readily that the above definition makes $M$ into an $R_{T}$-module. If $N$ is any $R$-submodule of $M$, then $N r=N$ for each $\mathrm{r} \in R \backslash I$. Hence $N r^{-1}=N$ and in general $N R_{I}=N$. So $N$ is an $R_{I}$-submodule of $M$. Obviously $R_{T}$-submodules are $R$-submodules, and the lemma follows.

Theorem 4. Let I be a maximal ideal of the duo ring $R$. The following are equivalent:

(1) $R_{I}$ is Artinian and the I-component of 0 is the right Icomponent of 0 .

(2) $E(R / I)$ has finite length.

(3) $E(R / I)$ is Noetherian.

Proof. Suppose that (1) is true and let $J$ be the right component of 0 . Then $J \subseteq I$ and $I / J$ is contained in the maximal ideal of $R_{I}$. But $R_{I}$ is Artinian, so $(I / J)^{n}=0$ for some integer $n$. Hence $I^{n} \subseteq J$. Let $t \in I^{n}$ and select $r \in R \backslash I$ such that $t r=0$. Then let $E=E(R / I)$ and obtain that $(E t) r=0$. Right multiplication of elements of $E$ by $r$ is a one-to-one map of $E$ into $E$. For if $0 \neq m \in E$ and $m r=0$, then select $s \in R$ such that $0 \neq m s \in R / I$ (possible since $E$ is an essential extension of $R / I$ ) and obtain that $0=m r=m r R=m R r$ and $m s r=$ 0 . This is a contradiction, since $R / I$ is a division ring. It follows from $(E t) r=0$ that $E t=0$. Consequently $t \in E \cdot 0$. . Therefore $I^{n} \subseteq$ $E \cdot 0$. Hence if $N$ is a submodule of $E$, then $N I^{n}=0$ for some integer $n$. Induct on $n$ to show that $N r=N$ for all $r \in R \backslash I$. If $n=$ 
1 , then $N$ is an $(R / I)$-vector space and it is clear that $N r=N$. If the assertion is true for integers less than $n$ and $N I^{n}=0$, then $N I$ is a submodule of $M$ such that $(N I) I^{n-1}=0$, so $N I r=N I$ by induction. Select $s \in R$ such that $1-s r \in I$ (possible since $R / I$ is a division ring). Then if $n \in N$, we have $n-n s r \in N I=N I r$, say $n-n s r=$ $n^{\prime} r, n^{\prime} \in N$. We obtain that $n=\left(n^{\prime}+n s\right) r \in N r$ and hence $N=N r$. It follows that $N=N r$ for all submodules $N$ of $E$ and $r \in R \backslash I$. Apply Lemma 2 to $E$ and obtain that $E$ is an $R_{I}$-module with the same submodule lattice as the $R$-module $E$. Let $L=I R_{I}$ be the maximal ideal of $R_{I}$ and we obtain that $E L^{n}=0$. Form the chain $R_{I} \supseteqq L \cong$ $\cdots \supseteqq L^{n}=0$ and set $E_{i}=\left\{x \in E \mid x L^{i}=0\right\}$ for $i=1, \cdots, n$. Then $E=$ $E_{n}$ and $E_{i+1} / E_{i}$ is isomorphic to a submodule of $\operatorname{Hom}_{R_{I}}\left(L^{i} / L^{i+1}, E\right)$ by Lemma 1 of [6, p. 373]. Now any image of $L^{i} / L^{i+1}$ is annihilated by $L$. Furthermore if $m \in E \backslash(R / I)$, then $m I \neq 0$ (else $m R$ is irreducible and meets $(R / I)$ trivially). Also $R / I \cong R_{I} / L$. Hence

$$
\operatorname{Hom}_{R_{I}}\left(L^{i} / L^{i+1}, E\right)=\operatorname{Hom}_{\left(R_{I} / L\right)}\left(L^{i} / L^{i+1}, R_{I} / L\right),
$$

which is just the dual space of $L^{i} / L^{i+1}$. Since $R_{I}$ is Artinian, $L^{i} / L^{i+1}$ is finite dimensional and so $E_{i+1} / E_{i}$ is Artinian. It follows that $E$ is an finite $R_{I}$-module and therefore a finite $R$-module. So (1) implies (2).

The equivalence of conditions (2) and (3) follows from Theorem 3.

Finally suppose that (2) is true. As above, let $J$ be the right $I$-component of $0, E=E(R / I)$ and $K=E \cdot .0 \subseteq I$. Since $E$ is finite, it is certainly Noetherian. We showed in the last part of the proof of Theorem 3 that if $E(R / I)$ is Noetherian, then $I^{n} \subseteq K$ for some positive integer $n$. If $x$ is any element of $R$ such that $x \cdot 0 \subseteq I$, then $x \notin K$ by Lemma 6 of $[6, \mathrm{p}$. 377] (simply lift the composition of maps $x R \rightarrow$ $R /(x \cdot 0) \rightarrow R / I$ to a map $R \rightarrow E)$. Consequently if $x \in K$, then there is an element $r \in R \backslash I$ such that $x r=0$. Hence $x$ belongs to $J$, the right $I$-component of 0 . Certainly $J$ is contained in the $I$-component of 0 , which we denote by $T$. Thus $I^{n} \subseteq K \subseteq J \subseteq T$. Then $R / T$ is a local ring with nilpotent ideal $I / T$. Consequently elements of $(R / T) \backslash$ $(I / T)$ are already units in $R / T$ and $R_{I}=R / T$. Also $R / T \cong(R / K) /(T / K)$, which is Artinian since $R / K$ is Artinian. Hence $R_{I}$ is Artinian. We now obtain, exactly as in the proof that (1) implies (2), that $N r=N$ for every submodule $N$ of $E$ and $r \in R \backslash I$. Consequently if $x \in T$, say $a x b=0$ for $a, b \in R \backslash I$, then $(E a x) b=0$. Therefore $0=(E a) x=E x$ and $x \in K=E \cdot 0$. It follows that $K=J=T$, which completes the proof that (2) implies (1).

REMARK 3. We have not been able to decide whether or not condition (1) of Theorem 4 may be replaced by the apparently weaker condition that $R_{I}$ is Artinian. In any case the condition that right $I$-component equal $I$-component is vacuous if $R$ is commutative. 


\section{REFERENCES}

1. M. Auslander and O. Goldman, Maximal orders, Trans. Amer. Math. Soc., 97 (1960), $1-24$.

2. E. H. Feller, Properties of primary noncommutative rings, Trans. Amer. Math. Soc., 89 (1958), 79-91.

3. J. Lambek, Lectures on Rings and Modules, Blaisdell Publishing Company, Waltham, Mass. 1966.

4. G. Maury, Charactérisation des ordres maximaux, C. R. Acad. Sc. Paris, 269 (1969), Ser. A, 993-996.

5. E. Matlis, Injective modules over Noetherian rings, Pacific J. Math., 8 (1958), 511528.

6. A. Rosenberg and D. Zelinsky, Finiteness of the injective hull, Math. Zeitschrift, 70 (1959), 372-380.

7. G. Thierrin, On duo rings, Canad. Math. Bull., 3 (1960), 167-172.

Received April 12, 1971.

The University of Nebraska 


\section{PACIFIC JOURNAL OF MATHEMATICS}

\section{EDITORS}

\section{H. SAMELSON}

Stanford University

Stanford, California 94305

\section{R. HOBBY}

University of Washington Seattle, Washington 98105

\section{J. DugundJI}

Department of Mathematics University of Southern California Los Angeles, California 90007

RICHARD ARENS

University of California Los Angeles, California 90024

\section{ASSOCIATE EDITORS}
E. F. BECKENBACH
B. H. Neumann
F. WoLF
K. YoSHIDA

\section{SUPPORTING INSTITUTIONS}

UNIVERSITY OF BRITISH COLUMBIA CALIFORNIA INSTITUTE OF TECHNOLOGY

UNIVERSITY OF CALIFORNIA

MONTANA STATE UNIVERSITY

UNIVERSITY OF NEVADA

NEW MEXICO STATE UNIVERSITY

OREGON STATE UNIVERSITY

UNIVERSITY OF OREGON

OSAKA UNIVERSITY

\author{
UNIVERSITY OF SOUTHERN CALIFORNIA \\ STANFORD UNIVERSITY \\ UNIVERSITY OF TOKYO \\ UNIVERSITY OF UTAH \\ WASHINGTON STATE UNIVERSITY \\ UNIVERSITY OF WASHINGTON \\ * * * * \\ AMERICAN MATHEMATICAL SOCIETY \\ NAVAL WEAPONS CENTER
}

The Supporting Institutions listed above contribute to the cost of publication of this Journal, but they are not owners or publishers and have no responsibility for its content or policies.

Mathematical papers intended for publication in the Pacific Journal of Mathematics should be in typed form or offset-reproduced, (not dittoed), double spaced with large margins. Underline Greek letters in red, German in green, and script in blue. The first paragraph or two must be capable of being used separately as a synopsis of the entire paper. The editorial "we" must not be used in the synopsis, and items of the bibliography should not be cited there unless absolutely necessary, in which case they must be identified by author and Journal, rather than by item number. Manuscripts, in duplicate if possible, may be sent to any one of the four editors. Please classify according to the scheme of Math. Rev. Index. to Vol. 39. All other communications to the editors should be addressed to the managing editor, Richard Arens, University of California, Los Angeles, California, 90024.

50 reprints are provided free for each article; additional copies may be obtained at cost in multiples of 50 .

The Pacific Journal of Mathematics is published monthly. Effective with Volume 16 the price per volume (3 numbers) is $\$ 8.00$; single issues, $\$ 3.00$. Special price for current issues to individual faculty members of supporting institutions and to individual members of the American Mathematical Society: $\$ 4.00$ per volume; single issues $\$ 1.50$. Back numbers are available.

Subscriptions, orders for back numbers, and changes of address should be sent to Pacific Journal of Mathematics, 103 Highland Boulevard, Berkeley, California, 94708.

PUBLISHED BY PACIFIC JOURNAL OF MATHEMATICS, A NON-PROFIT CORPORATION

Printed at Kokusai Bunken Insatsusha (International Academic Printing Co., Ltd.), 270, 3-chome Totsuka-cho, Shinjuku-ku, Tokyo 160, Japan. 


\section{Pacific Journal of Mathematics}

\section{Vol. 40, No. $3 \quad$ November, 1972}

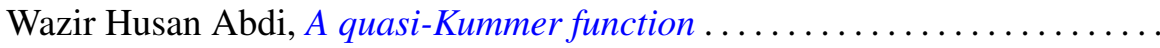

Vasily Cateforis, Minimal injective cogenerators for the class of modules of

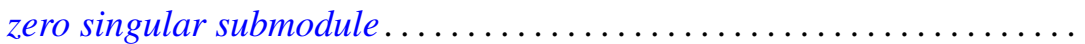

W. Wistar (William) Comfort and Anthony Wood Hager, Cardinality of

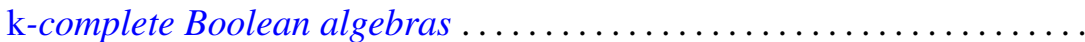

Richard Brian Darst and Gene Allen DeBoth, Norm convergence of martingales of Radon-Nikodym derivatives given a $\sigma$-lattice ..........

M. Edelstein and Anthony Charles Thompson, Some results on nearest points and support properties of convex sets in $c_{0} \ldots \ldots \ldots \ldots \ldots$

Richard Goodrick, Two bridge knots are alternating knots .

Jean-Pierre Gossez and Enrique José Lami Dozo, Some geometric properties related to the fixed point theory for nonexpansive mappings ..........

Dang Xuan Hong, Covering relations among lattice varieties .............

Carl Groos Jockusch, Jr. and Robert Irving Soare, Degrees of members of $\Pi_{1}^{0}$

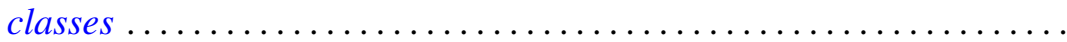

565

575

605

Leroy Milton Kelly and R. Rottenberg, Simple points in pseudoline

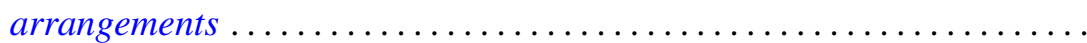

Joe Eckley Kirk, Jr., The uniformizing function for a class of Riemann surfaces....

Glenn Richard Luecke, Operators satisfying condition $\left(G_{1}\right)$ locally ... 629

T. S. Motzkin, On L $(S)$-tuples and l-pairs of matrices ... . .

Charles Estep Murley, The classification of certain classes of torsion free

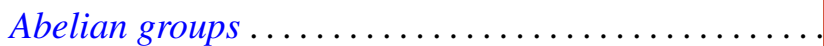

Louis D. Nel, Lattices of lower semi-continuous functions and associated topological spaces.

David Emroy Penney, II, Establishing isomorphism between tame prime

knots in $E^{3}$. . .

Daniel Rider, Functions which operate on $\mathscr{F} L_{p}(T), 1<p<2$

Thomas Stephen Shores, Injective modules over duo rings ...

Stephen Simons, A convergence theorem with boundary. .

703

Stephen Simons, Maximinimax, minimax, and antiminimax theorems and a

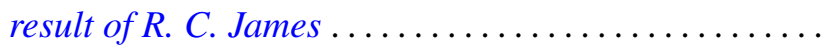

Stephen Simons, On Ptak's combinatorial lemma ........

Stuart A. Steinberg, Finitely-valued $f$-modules............

Pui-kei Wong, Integral inequalities of Wirtinger-type and fourth-order

elliptic differential inequalities .

Yen-Yi Wu, Completions of Boolean algebras with partially additive

operators ..................................

Phillip Lee Zenor, On spaces with regular $G_{\delta}$-diagonals . . . 